Type of the Paper: Article

\title{
Impact of Partial lockdown on Time-Dependent Effective Reproduction Number of COVID-19 infection in Ghana: Application of change point analysis
}

\author{
Joseph Armachie ${ }^{1,3^{*}}$, Agnes Adom-Konadu ${ }^{2}$, Morrison Asiamah ${ }^{1}$ and Duah Dwomoh ${ }^{3}$
}

1 Department of Electrom Microscopy and histopathology. Noguchi Memorial Institute For Medical Research, University of Ghana. Legon-Accra, Ghana 1; Armachiejoseph@gmail.com (JA), morrisonasiamah2012@gmail.com

2 Department of Mathematics and Statistics University of Cape Coast. Cape Coast, Ghana; agnes.donkor@ucc.edu.gh (AAK)

3 Department of Biostatistics, School of Public Health, University of Ghana, Legon-Accra, Ghana. duahdwomoh@ug.edu.gh (DD)

* Correspondence: duahdwomoh@ug.edu.gh, +233207861179 (DD)

\begin{abstract}
In 2020, the world was plagued with COVID-19 which was first identified in Wuhan, China in November 2019. During the early phase of the pandemic with no known treatment and WHO-approved vaccines, most government and public health officials were pushing for non-pharmaceutical intervention in trying to curb the spread of COVID-19. One such intervention is the restriction on movement to reduce social mixing. However, the economic cost of locking down cities especially in developing countries is unbearable as most of these countries are running economies that were on life support before the COVID-19 pandemic. We analyze the daily COVID-19 cases in Ghana to evaluate whether there was a significant change in transmission of COVID-19 after the partial lockdown of the two main cities (Kumasi and Accra) was lifted. The results showed that there was no increase in the transmissibility of COVID-19 after the partial lockdown was lifted. Contrary to the expectation that transmissibility will increase after lifting the partial lockdown, there was a reduction of the time-dependent reproduction ratio from 3.17 to 1.23 .
\end{abstract}

Keywords: COVID-19; Effective reproductive number; Lockdown.

\section{Introduction}

In December 2019, the world was faced with an emergence of a new pneumoniacausing virus that emerged from Wuhan a province in Hubei, China [1] which was later named severe acute respiratory syndrome coronavirus-2 (SARS-CoV-2) [2]. The virus was spreading through China and by January $20^{\text {th }}, 2020$, the virus had traveled outside of China to many countries including Taiwan, Thailand, Vietnam, Malaysia, Nepal, Sri Lanka, Cambodia, Japan, Singapore, Republic of Korea, United Arab Emirates, United States, Philippines, India, Australia, Canada, Finland, France, and Germany [3]. By 11 ${ }^{\text {th }}$ March 2020, the World Health Organization had declared that the world was in a COVID19 pandemic [4]. Despite efforts to curb the transmission of the virus, the virus had affected more than 10,000000 people around the globe with more than half a million death by the end of July 2020 [5]. Currently, there is no known treatment for COVID-19. This has left health care providers to rely on other therapeutic drugs which have been proven effective against other viruses exhibiting similar symptoms. Whereas there are different candidate vaccines at different stages of development [6] with some approved by FDA[7]. The recent emergence of the mutant strains of the COVID-19 virus in different countries including South Africa and the United Kingdom [8] and the subsequent spread of these 
strains to other countries makes the use of vaccines if available for the fight against COVID-19 more difficult as the vaccine may not be effective against the new strain.

One of the most important characteristics of the spread of infectious disease in mathematical modeling is the basic reproductive number $R_{o}$. The $R_{o}$ is defined as the average number of secondary infections caused by an infectious person in his/her entire infectious period [9]. This parameter quantifies the scale of an infection, whether there will be an epidemic that keeps on growing or an epidemic will die out in the long run. When available, the basic reproductive number can be useful for the estimation of the total number of individuals expected to be infected in an outbreak. Estimating the $R_{o}$ for an infectious disease quantifies the transmissibility of the disease and allows researchers and public health practitioners to have an indication of the long-term impact of the intervention on the infection spread due to the changes in $R_{o}$ resulting from the intervention. Ghana since recording its first COVID-19 case has put in different measures to curb the transmission of the virus. Some of these measures include enhanced hygiene protocols, social distancing at public places, partial lockdown, closure of borders [10]. These measures came with enormous discomfort to the citizens, reduced revenue to the government, and widening poverty gaps in the country. Measures to reduce social mixing such as the lockdown of cities and the closure of business centers have a tremendous consequence on developing economies like Ghana. The cost of feeding the vulnerable during the three weeks partial lock in the two most populous cities in Ghana cost over 52 million cedis according to the Finance Minister in the 2020 midyear review budget. One cannot demur the fact that, total or partial lockdown significantly reduces social mixing [11]. However, the impact of the reduced social mixing on the transmissibility of COVID-19 has not been well studied across Africa.

As the world including Ghana prepares to live in the new normal and relaxing the restrictions imposed to reduce social mixing, we analyze the daily count data from Ghana to evaluate the impact of the restriction of movement on the transmissibility of COVID19. We estimate the time-dependent reproductive number as a measure of transmissibility and investigate whether lifting the restriction increased the transmissibility of COVID-19 in Ghana.

\section{Methods}

\subsection{Data collection and Analysis Plan}

Daily recorded cases of COVID-19 infection in Ghana were obtained from the live statistics as reported by worldometer presented on its website [12]. The data were transferred into an excel file and were analyzed using $\mathrm{R}$. The Basic reproductive number describes the average number of secondary infections an infectious individual produces in his/her entire infectious life when in an entirely susceptible population. This parameter can be estimated using different approaches under different assumptions. In this research, we obtain the time-dependent reproductive number which is also known as an effective reproductive number using a likelihood approach from the daily count data. The effective reproductive number describes the average number of new infections produced by an infectious person in a population that is not fully susceptible. We also obtain the basic reproductive number using the maximum likelihood approach to evaluate the average transmissibility of COVID-19 in Ghana.

\subsection{Time-dependent estimate}

In estimating the time-dependent reproductive number, we employ the method discussed by [13]. Here, we give a general overview of the method used and refer the reader to [13] for a comprehensive overview. The outbreak of the epidemic is considered as a directed network where the nodes represent infection and edges represent transmission. A probability model is used to infer the infection network that underlies the epidemic 
curve at time $t$. The model is built on the assumption that infection occurred only in the reported cases

Let $\omega(\tau \mid \theta)$ be the probability density function of the generation interval where $\tau$ and $\theta$ are the generation time and the parameter that specifies the distribution respectively. The generation interval is the interval between the time when an individual is infected by an infector and the time when this infector was infected. Its distribution underpins estimates of the reproductive number and hence informs public health strategies [14]

Also, let $\mathcal{V}$ be a network of epidemic $\mathbf{t}$, then the probability of observing an epidemic $\mathbf{t}$ with network $\mathcal{V}$ and parameter $\theta$ for the generation time is given as

$$
L(\mathcal{V}, \theta \mid \mathbf{t})=\prod_{i=1}^{n-q} \omega\left(t_{i}-t_{v(i)} \mid \theta\right)
$$

$n$ and $q$ represent the number of reported cases and number of cases whose source of infection is outside of the population.

Here, the interest is to obtain the likelihood of sets of infection networks, which is achieved by summing the likelihood over networks in a set

$$
L(\mathcal{V}, \theta \mid \mathbf{t})=c \sum_{\mathcal{V}} \prod_{i=1}^{n-q} \omega\left(t_{i}-t_{v(i)} \mid \theta\right)=c \prod_{i=1}^{n-q} \sum_{j=1, j \neq i}^{j=n} \omega\left(t_{i}-t_{j} \mid \theta\right)
$$

Where $c$ is the weight and assumed to be constant since unobserved transmission event from case $j$ to case $i$ is independent on transmission from case $j$ to any other $k$ case.

For an infectious network in which an infection $l$ was as a result of $k$, the integrated likelihood is given by

$$
L\left(\mathcal{V}_{(k, l)}, \theta \mid \mathbf{t}\right)=c \omega\left(t_{i}-t_{j} \mid \theta\right) \prod_{i=1}^{n-q} \sum_{j=1, j \neq i}^{j=n} \omega\left(t_{i}-t_{j} \mid \theta\right)
$$

So that the likelihood of case $k$ was infected by $l$ is

$$
P_{(k, l)}=\frac{L\left(\mathcal{V}_{(k, l)}, \theta \mid \mathbf{t}\right)}{L(\mathcal{V}, \theta \mid \mathbf{t})}=\frac{\omega\left(t_{i}-t_{j} \mid \theta\right)}{\sum_{m=1, j \neq k}^{m=n} \omega\left(t_{k}-t_{m} \mid \theta\right)}
$$

The distribution of the effective reproduction number for case $l$ infecting others is

$$
R_{(l)} \sim \sum_{k}^{n-q} \text { Bernouli }\left[P_{(k, l)}\right]
$$

And the expectation is given as

$$
E\left(R_{(l)}\right)=\sum_{k}^{n-q} P_{(k, l)}
$$

The average daily reproduction number is obtained by computing the arithmetic mean of $R_{(l)}$ for all cases $l$ who show the first symptoms of illness on day $t$. We used the generation time estimate from $[15,16]$.

\section{Maximum Likelihood estimated}

Given the daily number of infections over the course of an epidemic, the likelihood function of the basic reproductive ratio is given by

$$
\mathcal{L}\left(\mathrm{R}_{0, \mathrm{p}}\right)=\prod_{\mathrm{t}=1}^{\mathrm{T}} \frac{\mathrm{e}^{-\mu_{\mathrm{t}}} \mu_{\mathrm{t}} \mathrm{N}_{\mathrm{t}}}{\mathrm{N}_{\mathrm{t}} !}
$$

where $\mu_{t}=R_{0} \sum_{j=1}^{\min (k, t)} N_{t-j} p_{j}, N_{t}$ represent the incident data, $p_{j}$ is the generation time with $\mathrm{j}=1, \ldots, \mathrm{k}, \mathrm{T}$ is the most recent time point of observation in the early epidemic 
growth stage of the disease [17]. The maximum likelihood estimate of $R_{0}$ is obtained by solving for the values for which $\mathcal{L}\left(\mathrm{R}_{0}, \mathrm{p}\right)$ is maximum.

\subsection{Changepoint analysis}

In this study, we used change point analysis to investigate whether there was a significant increase in the transmissibility of COVID-19 after the partial lockdown was lifted. Change point analysis, it can be viewed as investigating for a change in the statistical property of a sequence of observation. The statistical property may be the mean or the variance of the event under study and has been used in many fields [18, 19]. Let $y=$ $\left(y_{1}, y_{2}, y_{3}, \ldots, y_{n}\right)$ be series of sequential observations whose statistical property we wish to study for the existence of changes. A changepoint is said to occur if $\tau \in(1,2,3, \ldots, n-1)$ such that the statistical property $\left(y_{1}, y_{2}, y_{3}, \ldots, y_{\tau}\right)$ and $\left(y_{\tau+1}, y_{\tau+2}, y_{3}, \ldots, y_{n}\right)$ are significantly different. This can be extended to include multiple change points such that $\tau_{1: \mathrm{m}}=\left(\tau_{1}, \tau_{2}, \tau_{3},, \tau_{\mathrm{m}}\right)$ and each changepoint is an integer between 1 and $\mathrm{n}-1$.

In a changepoint analysis, a test statistic is developed so that the likelihood ratio method can be used to determine the presence of changepoint/s. This method requires the computation of the maximum likelihood under the null hypothesis that there is no change point and the alternative hypothesis that there are $m$ changepoints. The task is to minimize.

$$
\sum_{i=1}^{m+1} C\left(y_{\tau_{(\mathrm{i}-1+1)}: \tau_{\mathrm{m}}}\right)+\beta f(m)
$$

Where $C$ is a cost function such as the negative log-likelihood and $\beta f(m)$ is a penalty term for overfitting the number of change points. This changepoint analysis was conducted using the changepoint package in $\mathrm{R}$.

\section{Results}

Ghana recorded its first COVID-19 case in 12th March 2020 and since have been reporting daily cases which has been rising steadily. The daily count of new cases as was reported by the government of Ghana is presented in figure1. Following the recording of the first few cases and understudying the daily cases, the government closed all the borders of the country from human traffic including sea and air travel and in addition, announced a ban on social gatherings including churches and mosques. Subsequent to the closure of borders, there was a partial lockdown in the two main cities in Ghana namely Greater Accra and Greater Kumasi for two weeks which was extended by an additional one week taking effect from $30^{\text {th }}$ March 2020 . However, religious meetings and other social gatherings were still banned until July where they were opened to the public with strict 
adherence

to

social

distancing

protocol.

Daily count of Covid-19 cases

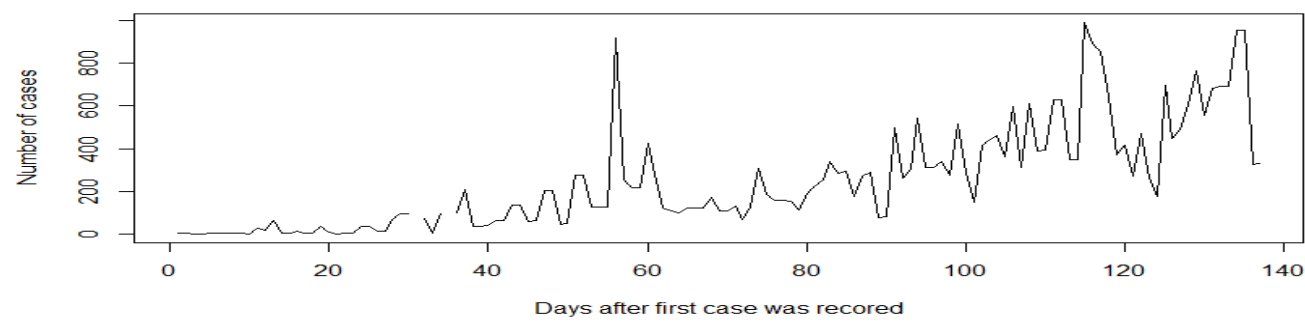

Cumulative covid-19 cases

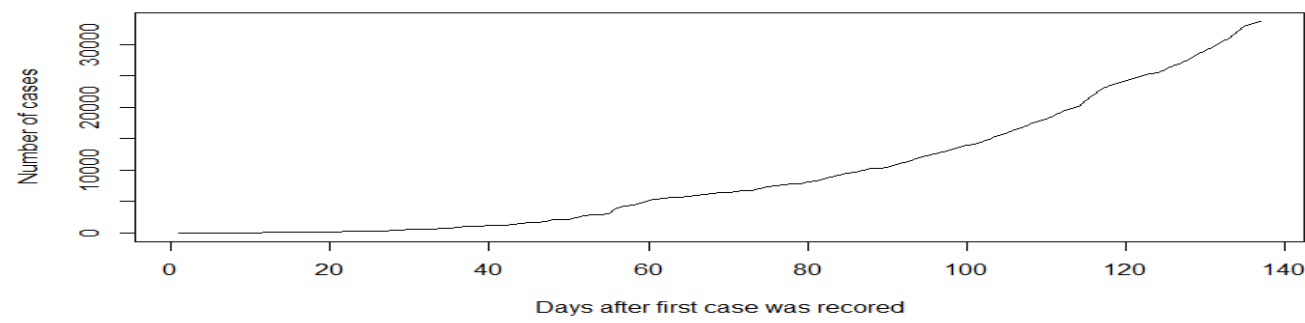

Figure 1. Graph A, the daily number of COVID-19 cases in Ghana. Graph B, the cumulative number of COVID-19 cases in Ghana.

The COVID-19 cases in Ghana have been growing steadily with spikes on $5^{\text {th }}$ May 2020 and $7^{\text {th }}$ June 2020. Here we estimate the basic reproductive number of the COVID19 cases in Ghana based on the maximum likelihood method. The Maximum likelihood estimate of basic reproductive number was 1.14 (95\% CI= $[1.12,1.16])$. A basic reproductive number of 1.14 indicates low transmissibility of the virus in Ghana. We further explored the basic reproductive number at different times during the epidemic where policy interventions were carried out and are represented in figure 2. The basic reproductive number was $3.13(95 \% \mathrm{CI}=[1.24,6.35])$ in the first nine days but later dropped to 1.14 $(95 \% \mathrm{CI}=[1.12,1.16])$ by the fourth month after the first case was recorded

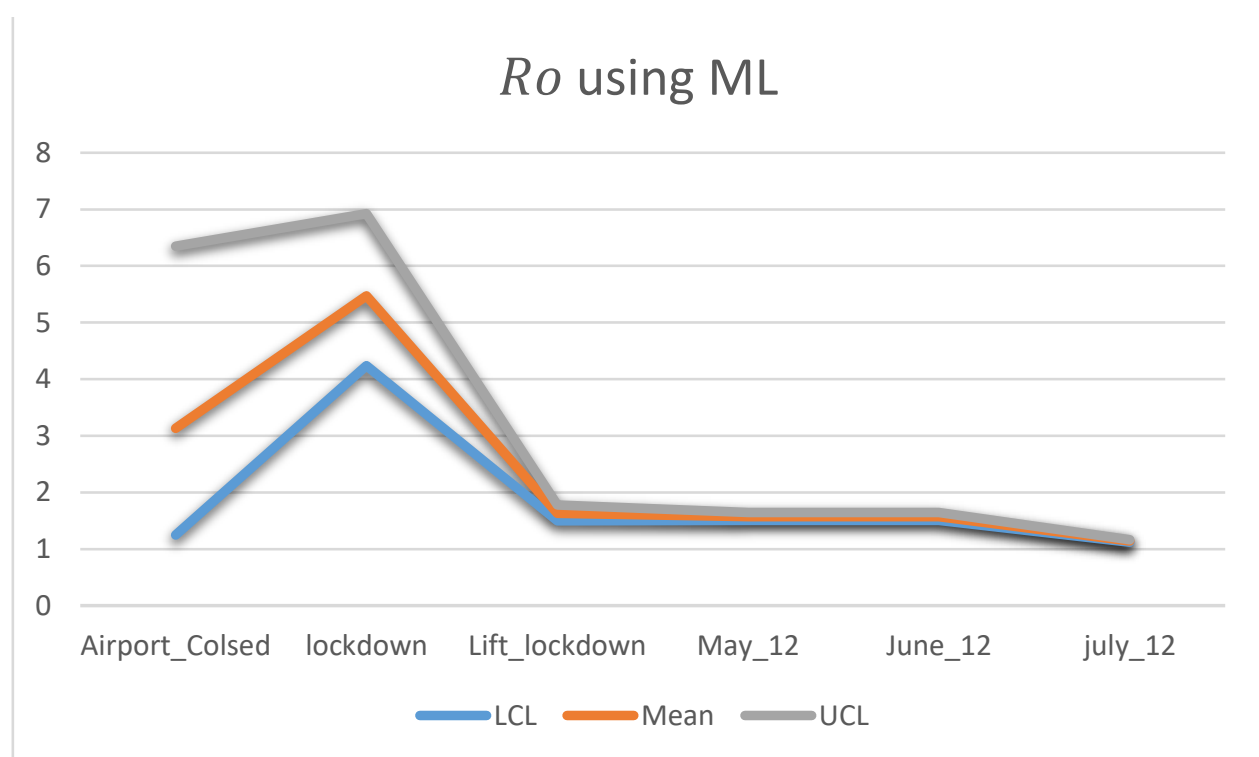

Figure 2. A graph showing the basic reproductive number and its corresponding confidence interval estimated using the maximum likelihood method at different time points where there was policy intervention.

We proceed to analyze the time dependent reproductive number. A graph of the time dependent basic reproductive number together with its $95 \%$ confidence interval is shown 
in figure 3. The time-dependent reproductive number has been changing with respect to time over the 137 days of data for which the analysis was conducted. An eyeball inspection shows that the time-dependent reproductive number was higher in the early stages of the epidemic and drops steadily as time elapses and later oscillates around the neighborhood of 1.17 .

Here we treat the time-dependent reproductive number as time series data and investigate the presence of change in the mean over time. Figure 3 represents the time-dependent reproductive number and dates when the social mixing policy was implemented by the government. We run the analysis to estimate the changepoints in the series of timedependent reproductive number using the AMOC method implemented in the changepoint package in $\mathrm{R}$. The results show that there was a shift in the average time-dependent reproductive number 9 days after recording the first case. The $R(t)$ averaged at 3.178124 within the first 9 days after the first COVID-19 case was recorded and dropped to 1.229314 thereafter.

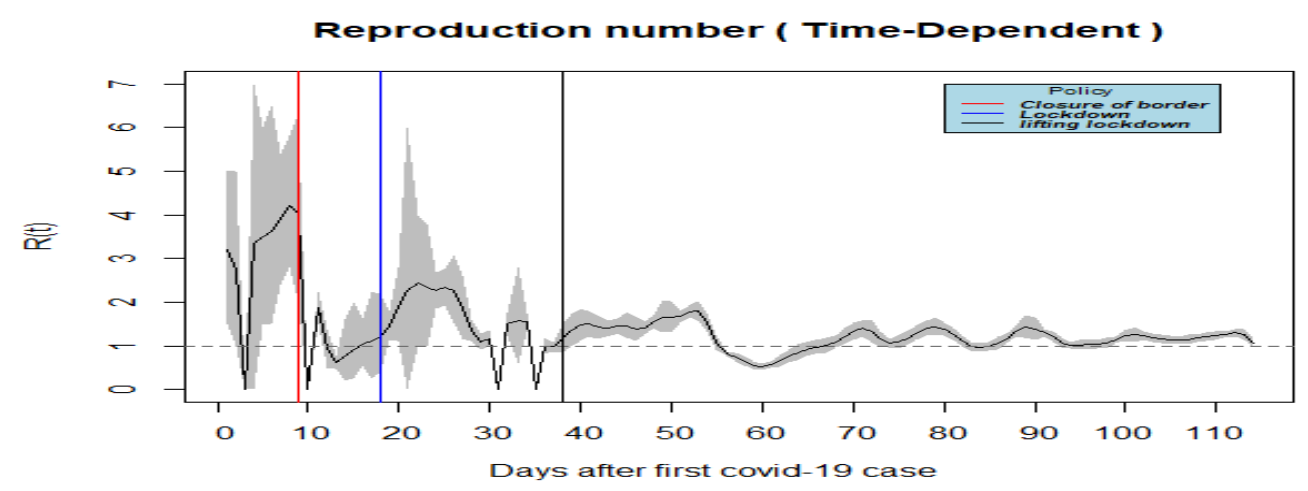

Change point analysis

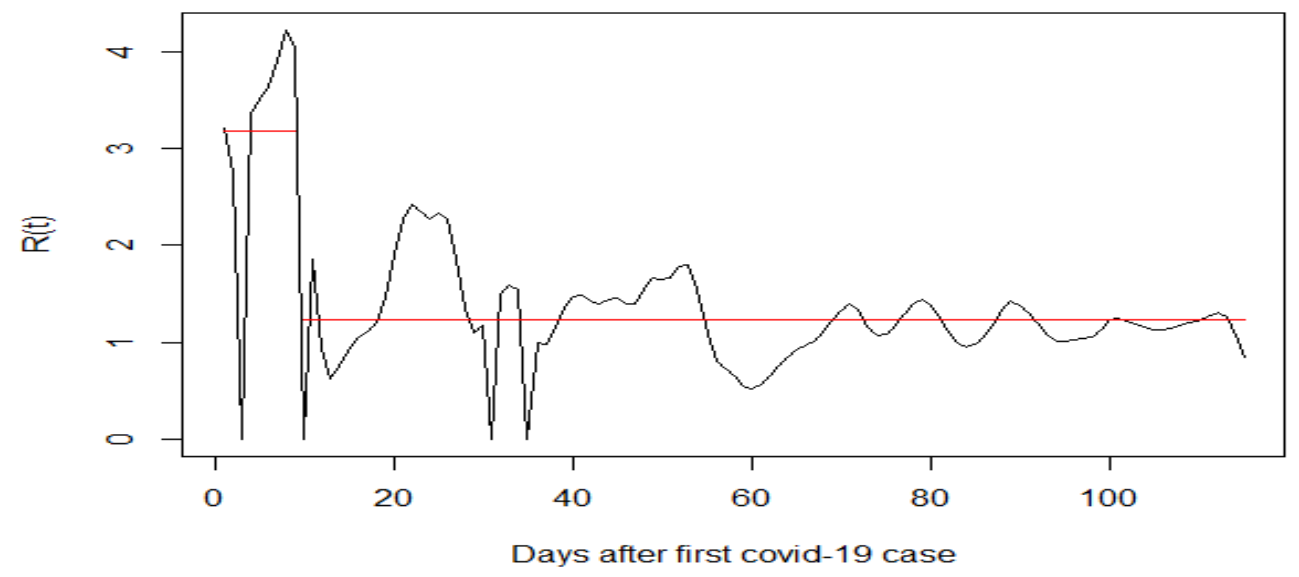

Figure 3. Graph A shows of the time-dependent reproductive number with its $95 \%$ confidence interval. Graph B shows the results from the Changepoint analysis where the red line shows the average time-dependent reproductive number.

We performed sensitivity analysis over a range of different choices of generation time distribution to investigate how the changes in the time-dependent reproductive number respond to the choice of the generation time distribution. Table 1 shows the number of changepoint and its corresponding average daily reproductive number obtained from using each of the distributions.

Table 1. Sensitivity analysis of the choice of the distribution for the generation time.

\begin{tabular}{llll}
\hline Distribution & Number of changepoint & Position of changepoint & $\mathrm{R}(\mathrm{t})$ \\
\hline Gamma & 1 & 9 & $3.17 / 1.23$ \\
\hline
\end{tabular}




\begin{tabular}{llll}
\hline Weibull & 1 & 9 & $3.15 / 1.22$ \\
lognormal & 1 & 9 & $3.3 / 1.24$ \\
\hline
\end{tabular}

\section{Discussion}

We investigated the impact of social mixing on the transmissibility of COVID-19 cases in Ghana. We hypothesized that lifting the restriction on movement in the two most populous cities in Ghana would increase the transmissibility of COVID-19 thereby increasing the daily count of cases. By way of estimating the time-dependent reproductive number, we checked for changes in the daily reproductive number. The basic reproductive number obtained using the maximum likelihood method was 1.14. This indicates low transmissibility of COVID-19 in the country. A little effort such as isolation of infectious individuals or adherence to hygiene will reduce basic reproductive number to a number below 1 which is required to contain the virus. The time-dependent reproductive number was found to be having a lot of variation in the early stage of the epidemic and later became stable. The results from the analysis showed an average time-dependent reproductive number was 1.4 and a standard deviation of 0.80 with a general decline in the reproductive ratio over the course of the epidemic. We applied change point analysis on the time-dependent reproductive number over the course of the epidemic. We tested the hypothesis that there was no change in time-dependent reproductive number over the course of the epidemic even after the partial lockdown was lifted. With the assumption that lifting the partial lockdown will increase social mixing and hence increase COVID-19 transmissibility, we expected the average reproductive number to increase when the partial lockdown was lifted. However, the analysis showed a decline in the daily reproductive number. There was a change in the average time-dependent reproductive number nine days after the first case of COVID-19 was recorded. The average time-dependent reproductive number for the first nine days of the epidemic was 3.17 which dropped to an average of 1.23 after nine days which is very close to the result obtained using the maximum likelihood method. We run a sensitivity analysis on the choice of the distribution for the generation time (gamma, Weibull, and lognormal) distribution. The analysis showed that the point in the course of the epidemic where there was a change in the average timedependent reproductive number was the same regardless of the type of distribution of the generation time used in the analysis Authors should discuss the results and how they can be interpreted from the perspective of previous studies and of the working hypotheses. The findings and their implications should be discussed in the broadest context possible. Future research directions may also be highlighted.

The results from this research have shown that there was no increase in transmissibility of COVID-19 after the partial lockdown was lifted in the two most populated cities in Ghana even though the cost for locking down these cities was overwhelming for the government. This may be due to non-compliance of the lockdown restriction due to the informal nature of the Ghanaian economy and hence its removal had no effect. Market places, an area that is overcrowded and busy was open to the public with a lot of people not observing social distancing during the lockdown period. Also, most of the areas known to be a hot spot of the COVID-19 epidemic in Ghana were low-income-earning areas in the city where most residents shared hygiene facilities such as washrooms. This could further explain why the reduction in social mixing due to the partial lockdown did not translate to a reduction in transmissibility of COVID-19 in Ghana. This result has shown that restriction because of partial lockdown and its lack thereof may not have the intended impact on the transmissibility of COVID-19. Stakeholders and researchers may focus on other methods such as practicing improved hygiene, social distance, Isolation of infected persons, and use of face masks. 


\begin{abstract}
Author Contributions: DD and JA conceived the idea, designed the study and conducted the analysis. AAK and MA reviewed the manuscript and contributed to the write-up of the results and discussion sections of the manuscript.

Funding: Please add: This research received no external funding

Institutional Review Board Statement: Not applicable

Informed Consent Statement: Not applicable

Data Availability Statement: Data is freely available from the Ghana Health Service website (https://www.ghanahealthservice.org/COVID19/)

Acknowledgments: We duly acknowledge the Ghana Health Service Staff for making the data freely available online

Conflicts of Interest: The authors declare no conflict of interest
\end{abstract}

\title{
References
}

1. Wang C, Horby PW, Hayden FG, Gao GFJTL: A novel coronavirus outbreak of global health concern. 2020, 395(10223):470-473.

2. Gorbalenya AEJB: Severe acute respiratory syndrome-related coronavirus-The species and its viruses, a statement of the Coronavirus Study Group. 2020.

3. Rothan HA, Byrareddy SNJJoa: The epidemiology and pathogenesis of coronavirus disease (COVID-19) outbreak. 2020:102433.

4. Cucinotta D, Vanelli MJAb-mAP: WHO declares COVID-19 a pandemic. 2020, 91(1):157-160.

5. Organization WH: Coronavirus disease ( COVID-19): situation report, 165. 2020.

6. Le TT, Andreadakis Z, Kumar A, Román RG, Tollefsen S, Saville M, Mayhew SJNRDD: The COVID-19 vaccine development landscape. 2020.

7. Oliver SE, Gargano JW, Marin M, Wallace M, Curran KG, Chamberland M, McClung N, Campos-Outcalt D, Morgan RL, Mbaeyi SJM et al: The Advisory Committee on Immunization Practices' Interim

Recommendation for Use of Pfizer-BioNTech COVID-19 Vaccine-United States, December 2020. 2020, 69(50):1922.

8. Chen AT, Altschuler K, Zhan SH, Chan YA, Deverman BEJB: COVID-19 CG: Tracking SARS-CoV-2 mutations by locations and dates of interest. 2020.

9. Zhou T, Liu Q, Yang Z, Liao J, Yang K, Bai W, Lu X, Zhang WJJoEBM: Preliminary prediction of the basic reproduction number of the Wuhan novel coronavirus 2019-nCoV. 2020, 13(1):3-7.

10. Asante LA, Mills ROJAS: Exploring the Socio-Economic Impact of COVID-19 Pandemic in Marketplaces in Urban Ghana. 2020:0002039720943612.

11. Akim A-m, Ayivodji FJAaS: Interaction Effect of Lockdown with Economic and Fiscal Measures against COVID-19 on Social-Distancing Compliance: Evidence from Africa. 2020.

12. Coronavirus Cases [https://www.worldometers.info/coronavirus/country/ghana/]

13. Wallinga J, Teunis PJAJoe: Different epidemic curves for severe acute respiratory syndrome reveal similar impacts of control measures. 2004, 160(6):509-516.

14. Champredon D, Dushoff JJPotRSBBS: Intrinsic and realized generation intervals in infectious-disease transmission. 2015, 282(1821):20152026.

15. Nishiura H, Linton NM, Akhmetzhanov ARJIjoid: Serial interval of novel coronavirus (COVID-19) infections. 2020.

16. Aghaali M, Kolifarhood G, Nikbakht R, Mozafar Saadati H, Hashemi Nazari SSJT, diseases e: Estimation of the serial interval and basic reproduction number of COVID-19 in Qom, Iran, and three other countries: A data-driven analysis in the early phase of the outbreak. 2020. 
17. Forsberg White L, Pagano MJSim: A likelihood-based method for real-time estimation of the serial interval and reproductive number of an epidemic. 2008, 27(16):2999-3016.

18. Contal C, O'Quigley JJCs, analysis d: An application of changepoint methods in studying the effect of age on survival in breast cancer. 1999, 30(3):253-270.

19. Chen J, Gupta AK: Parametric statistical change point analysis: with applications to genetics, medicine, and finance: Springer Science \& Business Media; 2011. 